\title{
Relación entre las variables fisicoquímicas del agua y la dinámica sistémica del complejo cenagoso La Grande de Beté, Medio Atrato, Chocó, Colombia
}

\section{Relationship between the variable physics and chemistry of the water and the system dynamic of complejo cenagoso La Grande de Beté, Medio Atrato, Chocó, Colombia}

\author{
Giovanny Ramírez-Moreno, MSc (c) ${ }^{1}$, Lady Vargas Porras, Ing Amb ${ }^{2}$
}

\section{RESUMEN}

La ciénaga Grande de Beté priorizada para este estudio, es una ciénaga principal de tipo 1, conectada directamente con una fuente hídrica principal (río Atrato) a través del caño Torrobón, de ahí que la composición de sus aguas se encuentre influida por la dinámica del río Atrato y sus afluentes. La toma de muestras desde las zonas riverinas del humedal, pasando por el espejo de agua, las aguas de caño y la zona de conexión entre dicho caño y el río Atrato, y el posterior análisis de parámetros como nitritos, nitratos, fosfatos, sulfatos, alcalinidad, acidez, dureza, sólidos totales, sólidos disueltos y sólidos suspendidos demuestran que estas zonas contienen la información que permite describir muchos aspectos de la dinámica hídrica y biológica del ecosistema en estudio. Dicha dinámica física y química al interior de la ciénaga mostró que gran parte de la materia orgánica de la misma, es aportada por el material de arrastre de las llanuras aluviales del río Atrato y se encuentra disuelta en el agua por procesos de descomposición, y que su calidad presenta niveles que comparados con estándares internacionales permiten el desarrollo óptimo de la biota acuática típica de este tipo de sistemas. El presente estudio es una aproximación al conocimiento no sólo de los humedales del medio Atrato sino un análisis de la relación existente entre ecosistemas lenticos y loticos interconectados.

Palabras clave: Ciénaga; Dinámica; Caños; Variables fisicoquímicas; Medio Atrato.

\begin{abstract}
Cénaga Grande de Beté prioritized for this study is a major swamp type 1, connected directly to the main water source (Atrato rivero), through the Caño Torrobón, hence the composition of its waters is influenced by the dynamics of Atrato river and its tributaries. Samples from the riverine areas of the wetland, through the mirror of water, water caño and the connection area between the caño and the Atrato river, and further analysis of parameters such as nitrites, nitrates, phosphates, sulphates, alkalinity, acidity, hardness, total solids, dissolved solids and suspended solids show that these areas contain information that can describe many aspects of biological water dynamics and ecosystem under study. The physical and chemical dynamics within the Ciénaga showed that much of the organic matter of the same material is provided by the drag of the Atrato river uality levels compared to international standards that allow the optimal development of aquatic biota typical of such systems. This study is
\end{abstract}

1. Investigador principal, Componente Ecosistémico, Instituto de Investigaciones Ambientales del Pacífico, Quibdó, Chocó, Colombia. Programa de Maestría en Ciencias Biológicas, Universidad Nacional de Colombia, Instituto de Ciencias Naturales, Bogotá, Colombia.

e-mail:gramirezm@unal.edu.co gramirez@iiap.org.co

2. Profesional responsable, Capítulo Aguas Proyecto Inventario Priorización y Caracterización Ambiental de los Humedales del Medio Atrato, Chocó, Colombia.

e-mail: lady8181@hotmail.com

Recibido: agosto 26, 2008

Aceptado: septiembre 9, 2008 
an approach to knowledge not only of the Midio Atrato wetlands but an analysis of the relationship between lentic and lotic ecosystems interconnected.

Keywords: Ciénaga; Dynamic, Caños; Variables physicochemical; Medio Atrato

\section{INTRODUCCIÓN}

Como resultado de una amplia revisión bibliográfica de toda la información existente relacionada con el análisis de parámetros fisicoquímicas en aguas de las ciénagas del municipio del Medio Atrato, Chocó, Colombia, se encontró que no existen datos de estudios de este tipo realizados directamente en este municipio. No obstante, se encontraron datos de trabajos académicos a nivel de tesis de grado (Universidad Tecnológica del Chocó) que reportan resultados obtenidos de variables fisicoquímicas en la zona media de la cuenca del río Atrato y en dos humedales del municipio de Quibdó que pertenecen a su vez a dicha zona.

Es así como en la margen derecha de la zona media del río Atrato se han medido variables como temperatura del agua, conductividad, turbiedad, $\mathrm{pH}$, oxígeno disuelto, alcalinidad, dióxido carbónico y nutrientes como nitritos, amonio y fosfatos (Tabla 1).

También se han reportado estudios en las ciénagas La Grande (corregimiento Loma de Belén, Quibdó, Chocó) y Plaza Seca (corregimiento Sanceno, Quibdó, Chocó), ambas ubicadas en la misma zona. Las variables fisicoquímicas analizadas y los resultados obtenidos en estas ciénagas aparecen en la Tabla 2.

Es de anotar que estos reportes se han obtenido como resultado de estudios a nivel académico con objetivos que no buscan analizar el estado general de los ecosistemas asociados con las aguas sino relacionarlos con la presencia y comportamiento de algunas especies en estudio, pero también es un acercamiento a los datos que se podrían encontrar en el proyecto planteado.

\section{METODOLOGÍA}

Área de estudio. Los ecosistemas cenagosos se conciben como flujos de energía representados en las interacciones de los organismos que habitan los diferentes estratos de la columna de agua y en las conexiones que presentan con otros sistemas acuáticos. La ciénaga Grande de Beté priorizada para este estudio es una ciénaga principal o de tipo 1 , es decir que se conecta directamente a una fuente hídrica principal (río) a través de un caño, de ahí que la composición de sus aguas se encuentre influida por la dinámica del río Atrato y sus afluentes. Se encuentra inmersa en todo un sistema hídrico donde el río es el eje principal y determinante junto con las condiciones climáticas en los cambios de nivel y direcciones de flujo que presenta. Cuenta con un caño bien definido denominado Amé pero a su vez se puede ingresar a ella por el caño Torrobón, que se conecta con otras ciénagas de la zona. El área de estudio para el componente agua abarca desde las zonas riverinas del humedal, pasando por el espejo de agua, las aguas de caño y la zona de conexión entre dicho caño y el río Atrato; estas zonas contienen la información que permite describir muchos aspectos de la dinámica hídrica y biológica del ecosistema en estudio.

Muestreo. La etapa de muestreo se llevó a cabo en el mes de noviembre durante la temporada de inundación de la zona; se midió el pH del agua in situ y se tomaron muestras para análisis en el laboratorio de nitritos, nitratos, fosfatos, sulfatos, alcalinidad, acidez, dureza, sólidos totales, sólidos disueltos y sólidos suspendidos; ambas actividades se realizaron georeferenciando los puntos escogidos.

Se definieron 5 puntos de muestreo con el fin de tener resultados por cada zona de la ciénaga, con el objeto de entender la dinámica del ecosistema y el flujo de los nutrientes y energía que presenta en relación con todo el sistema hídrico al que hace parte. En la Figura 1 se muestran los 5 puntos mencionados.

Mediciones in situ Se utilizó un pHmetro y un GPS para realizar mediciones georeferenciadas in situ del $\mathrm{pH}$ del agua en las zonas de muestreo determinadas.

Mediciones en laboratorio. Se tomaron muestras de agua para análisis en laboratorio en los 5 puntos definidos, que permitieran comparar los resultados entre la composición del agua de las diferentes zonas de la ciénagas y a su vez

Tabla 1

\section{Características fisicoquímicas de las aguas de la parte media de la cuenca del río Atrato}

\begin{tabular}{lc}
\hline Variable & Valor promedio \\
\hline Temperatura del agua & $25.6^{\circ} \mathrm{C}$ \\
Conductividad & $0.03 \mathrm{mS} / \mathrm{cm}$ \\
Turbiedad & $97 \mathrm{UNT}$ \\
Ph & $7.6 \mathrm{u} \mathrm{Ph}$ \\
Oxígeno disuelto & $5.7 \mathrm{mg} / / \mathrm{I}_{2}$ \\
Dióxido carbónico & $4.1 \mathrm{mg} / \mathrm{l} \mathrm{CO}_{2}$ \\
Alcalinidad & $64 \mathrm{mg} / \mathrm{l} \mathrm{CACO}$ \\
Amonio & $0.13 \mathrm{mg} / \mathrm{l} \mathrm{NH}_{4}$ \\
Nitritos & $0.1 \mathrm{mg} / \mathrm{I} \mathrm{NO}_{2}$ \\
Fosfatos & $0.2 \mathrm{mg} / \mathrm{PO}_{4}$ \\
\hline
\end{tabular}

Fuente: Torres Allin, 2002 


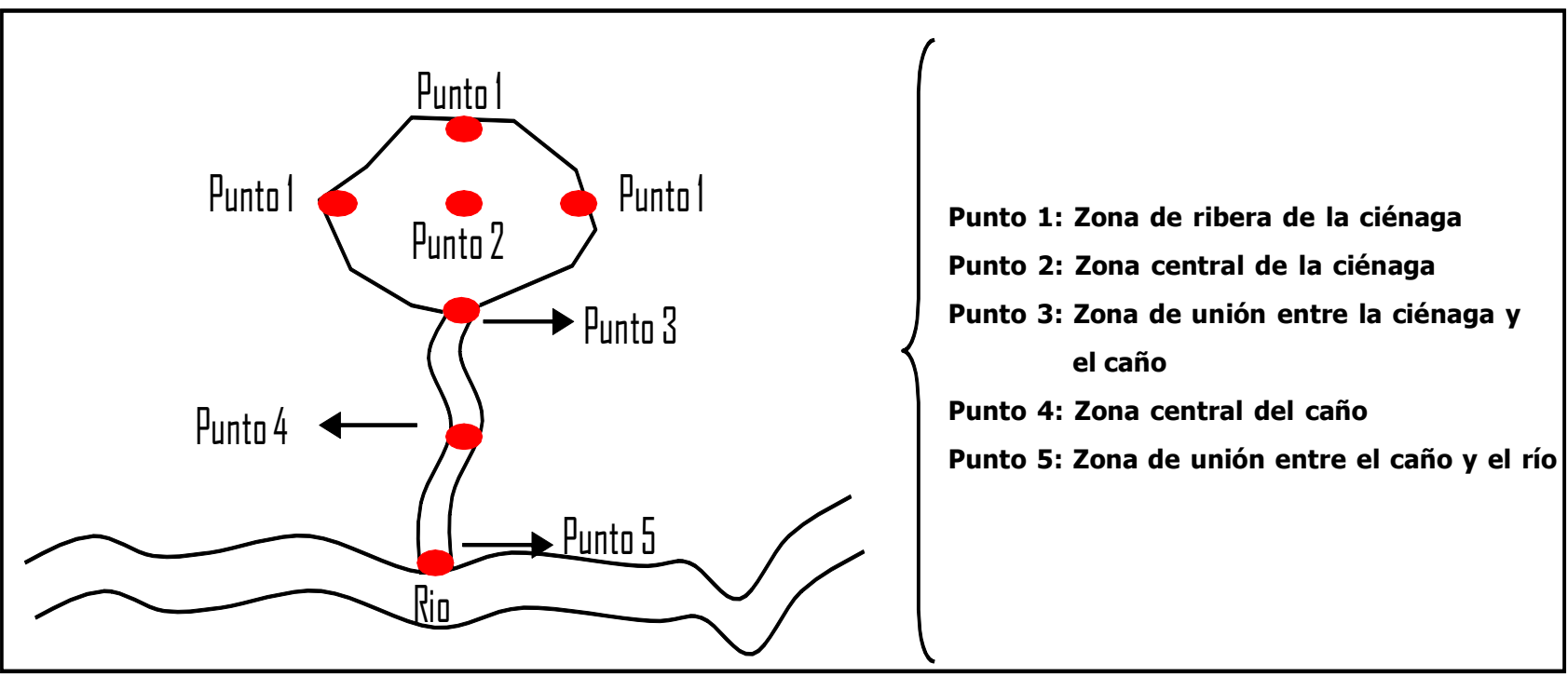

Figura 1. Diagrama idealizado de la zonificación de muestreo

Tabla 2

Características fisicoquímicas de las aguas de dos ciénagas del municipio de Quibdó, zona media de la cuenca del río Atrato

\begin{tabular}{|c|c|c|c|}
\hline \multicolumn{2}{|c|}{ Ciénaga La Grande de Quibdó } & \multicolumn{2}{|c|}{ Ciénaga Plaza Seca } \\
\hline Variable & Valor promedio & Variable & Valor promedio \\
\hline Temperatura del agua & $26.6^{\circ} \mathrm{C}$ & Temperatura del agua & $25.9^{\circ} \mathrm{C}$ \\
\hline Conductividad & $0.51 \mu \mathrm{s} / \mathrm{cm}$ & Conductividad & $26 \mu \mathrm{s} / \mathrm{cm}$ \\
\hline $\mathrm{Ph}$ & 5.6 uph & Turbiedad & $18.3 \mathrm{mg} / \mathrm{l}$ \\
\hline Oxígeno disuelto od & $2.1 \mathrm{mg} / \mathrm{l} \mathrm{O}_{2}$ & $\mathrm{Ph}$ & $6.3 \mathrm{u} \mathrm{ph}$ \\
\hline Alcalinidad & $3.7 \mathrm{mg} / \mathrm{l} \mathrm{CACO}_{3}$ & Dióxido carbónico & $5.6 \mathrm{mmol} / \mathrm{l} \mathrm{CO}$ \\
\hline Amonio & $0.2 \mathrm{mg} / \mathrm{l} \mathrm{NH}$ & Alcalinidad & $30.8 \mathrm{mg} / \mathrm{l} \mathrm{CACO}{ }_{3}$ \\
\hline Nitritos & $17 \mathrm{mg} / \mathrm{l} \mathrm{NO}$ & Amonio & $1 \mathrm{mg} / \mathrm{l} \mathrm{NH}{ }_{4}$ \\
\hline Nitratos & $50.2 \mathrm{mg} / \mathrm{l} \mathrm{NO}_{3}$ & Nitritos & $0.25 \mathrm{mg} / \mathrm{l} \mathrm{NO}$ \\
\hline Fosfatos & $0.07 \mathrm{mg} / \mathrm{l} \mathrm{PO}$ & Fosfatos & $0.5 \mathrm{mg} / \mathrm{l} \mathrm{PO}$ \\
\hline Profundidad & $2.65 \mathrm{~m}$ & Profundidad & $248.5 \mathrm{~cm}$ \\
\hline
\end{tabular}

Fuente: Cuesta \& Cuesta, 2001, Maturana \& Cuesta, 2002

relacionarla con la observación directa de los cambios de flujo de agua entre río- ciénaga, el estado general del ecosistemas y el componente biológico, facilitando la comprensión de la dinámica general del ecosistema.

Se realizó un muestreo simple o puntual en donde se colectaron muestras teniendo en cuenta el parámetro a analizar, el tipo de recipiente, los métodos de preservación y el tiempo transcurrido entre la toma de la muestra y el análisis. Los análisis de las mismas se efectuaron en el Laboratorio
Ambiental Certificado de la Corporación Autónoma para el Desarrollo Sostenible delChocó (CODECHOCO), de acuerdo con las metodologías establecidas, las cuales se especifican en la Tabla 3.

Como herramienta metodológica de comparación de parámetros de calidad de agua, los resultados obtenidos por cada parámetro fisicoquímico analizado en el agua de la ciénaga Grande de Beté se comparó con estándares internacionales de calidad de agua para la preservación de la biota 
Tabla 3

Metodologías empleadas para análisis de muestras de aguas $(\mathrm{mg} / \mathrm{I})$

\begin{tabular}{ll}
\hline \multicolumn{1}{c}{ Parámetro } & \multicolumn{1}{c}{ Método } \\
\hline Alcalinidad total & Titulométrico \\
\hline Nitritos & Espectofotométrico \\
Dureza total & Titulométrico \\
Sólidos totales & Gravimétrico \\
Nitratos & Reducción con cadmio \\
Sólidos disueltos & Gravimétrico \\
\hline Sulfatos & Gravimétrico \\
Sólidos suspendidos totales & Gravimétrico \\
Acidez & Titulométrico \\
Fosfatos & Ácido ascórbico \\
\hline
\end{tabular}

Fuente: CODECHOCÓ

acuática, teniendo en cuenta que el concepto de calidad de las aguas naturales está ligado con su uso y que numerosos países como EUA, Canadá, Chile, Uruguay, Argentina, entre otros, han incorporado el concepto de usos designados para establecer los criterios de evaluación y protección de la calidad de las aguas naturales. Para evaluar la calidad de las aguas, estos países comparan sus resultados de monitoreo con estándares de calidad los cuales combinan los usos designados (natación, pesca, etc), con criterios para proteger esos usos (como umbrales específicos para sustancias químicas, los cuales no pueden ser excedidos) y una política antidegradación con el propósito de que las aguas que cumplen los estándares no sean degradadas de su condición actual.
De acuerdo con la EPA [Enviromental Protection Agency, Agencia de Protección Ambiental, USA, Water Quality Standards (WQS), Patrones de Calidad de Agua], los estándares de calidad de agua son reglas que especifican la calidad que un determinado Estado desea alcanzar o mantener en sus aguas superficiales (lagos, arroyos, ríos, humedales), para proteger la calidad de los recursos acuáticos existentes de su degradación, y reglamentar las descargas puntuales y no puntuales. Es así como existen estándares internacionales de calidad adoptados para proteger a los peces y toda la vida acuática. A continuación se presentan en las Tablas 4, 5 y 6 los estándares internacionales de calidad de agua para la preservación de la vida acuática con los cuales se compararon los resultados obtenidos de parámetros físicoquímicos en el presente estudio para la Ciénaga Grande de Beté.

\section{RESULTADOSYDISCUSIÓN}

Resultados de análisis in situ. En la Tabla 7 se muestran los resultados obtenidos durante la medición in situ del pH en la ciénaga la Grande de Beté.

$\boldsymbol{p H}$. El pH en el agua se refiere a la concentración de iones $\mathrm{H}+$ que ésta tenga y está involucrado con la acidezy basicidad. Los valores de $\mathrm{pH}$ van de 0 a 14; cuando es 7 se dice que es neutro, menor a 7 es ácido y mayor es básico. Es un parámetro muy importante para muchos organismos, algunos de los cuales pueden ser sensibles a los cambios de $\mathrm{pH}$, otros en cambio bastante tolerantes.

En la Tabla 7 se observa que el $\mathrm{pH}$ es en general ácido para todas las zonas, presentándose aguas con $\mathrm{pH}$ más ácidos en las zonas de rivera y de espejo de agua de la ciénaga y valores cercanos a pH neutro en las zonas de caño y de influencia del río. Esto se explica teniendo en cuenta que el flujo de agua se comporta en dirección río-ciénaga, llevando con él gran

Tabla 4

Parámetros indicadores de calidad de agua para la preservación de la vida acuática en Panamá

\begin{tabular}{|c|c|c|c|}
\hline Uso & Parámetro & Valor aceptable & Efecto \\
\hline \multirow{4}{*}{ Vida acuática } & Nitritos & Menor de $0.6 \mathrm{mg} / \mathrm{l}$ & Produce enfermedades en peces. \\
\hline & Nitratos & Menor de 200mg/l & $\begin{array}{l}\text { Estimula el crecimiento de algas y plantas acuáticas que } \\
\text { pueden ocasionar una disminución del nivel de oxígeno. } \\
\text { También puede afectar la salud de los peces. }\end{array}$ \\
\hline & $\mathrm{pH}$ & 5.0 a 9.0 & $\begin{array}{l}\text { Fuera del rango indicado, puede afectar a los peces } \\
\text { dependiendo de la especie. }\end{array}$ \\
\hline & AmonÍaco & Menor de $1.2 \mathrm{mg} / \mathrm{l}$ & $\begin{array}{l}\text { Es tóxico para los peces dependiendo de la temperatura y el } \\
\mathrm{pH} \text {. }\end{array}$ \\
\hline
\end{tabular}

Fuente: Cooke, R. et al., 2001 


\section{Tabla 5 \\ Estándares de calidad de aguas para preservación de la biota acuática en Uruguay}

\begin{tabular}{lll}
\hline Variable & Unidad & Decreto $\mathbf{2 5 3 / 7 9}$ \\
\hline $\mathrm{pH}$ & & 6.5 a 8.5 \\
$\mathrm{NH3}$ & $\mathrm{microg} / \mathrm{l}$ & 20 \\
Fósforo total & $\mathrm{mg} / \mathrm{l}$ & 0.025 \\
\hline
\end{tabular}

Fuente: Ministerio de Vivienda, Ordenamiento territorial y Medio ambiente de Uruguay, 2007

Tabla 6

Listado de parámetros y niveles de concentración para preservar la biota acuática en Argentina

\begin{tabular}{ll}
\hline \multicolumn{1}{c}{ Parámetro } & \multicolumn{1}{c}{ Valores permisibles } \\
\hline $\mathrm{pH}$ & De 6 a 9.0 \\
Amoniaco & $\begin{array}{l}\text { La concentración no debe superar } \\
0.75 \mathrm{mg} / \mathrm{l}\end{array}$ \\
& $\begin{array}{l}\text { La concentración no debe superar } \\
\text { Nitratos }\end{array}$ \\
Sulfatos & $\begin{array}{l}\text { La concentración no debe superar } \\
500 \mathrm{mg} / \mathrm{I}\end{array}$ \\
\hline
\end{tabular}

Fuente: (Carrizo R. 2008)

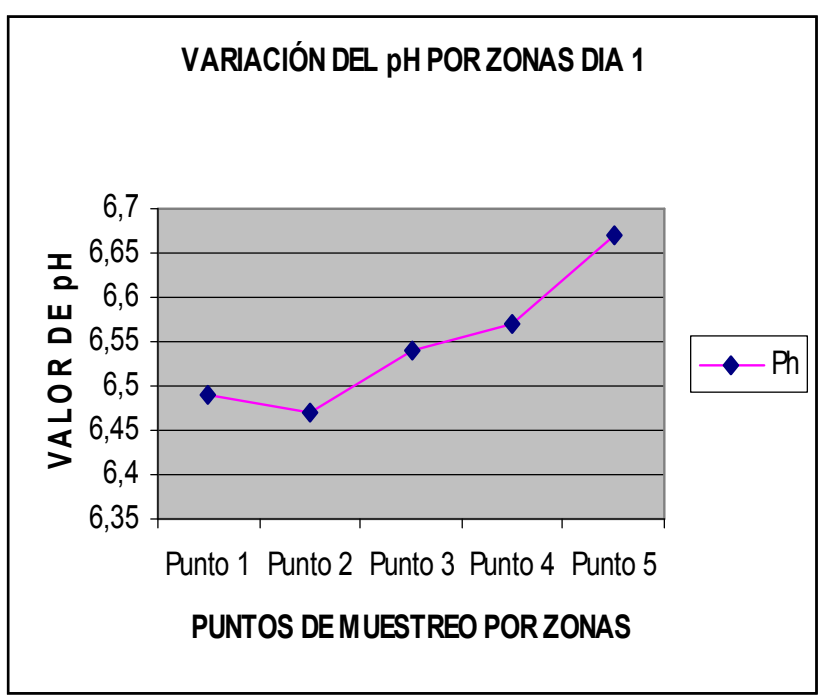

cantidad de sólidos suspendidos y material disuelto que aunque alcanzan a llegar en menores proporciones a la ciénaga debido al filtro ejercido por la morfología del suelo y la vegetación, ingresa una cantidad considerable, que inicia un proceso de descomposición al interior de la ciénaga que está relacionado directamente con la variación del $\mathrm{pH}$ del agua, de ahí que este parámetro disminuya espacialmente de acuerdo con la ubicación de la actividad de concentración y descomposición de materia orgánica, como son las zonas ribereñas y centrales de la ciénaga. La Gráfica 1 muestra la variación del $\mathrm{pH}$.

Resultados de mediciones en laboratorio. En la Tabla 8 se muestran los resultados de análisis en laboratorio obtenidos para las 5 zonas de muestreo definidas en campo.

Nutrientes. Los nutrientes (compuestos de nitrógeno, fósforo y azufre) determinan la productividad primaria en el medio acuático. Las concentraciones altas pueden producir eutroficación, aumentando poblaciones de algas y plantas acuáticas, produciendo efectos dañinos en el ecosistema (APHA-AWWA-WPCF, 1992).

El nitrógeno oxidado total es la suma de los nitritos y nitratos, constituye un nutriente esencial para muchos autótrofos fotosintéticos y en algunos casos ha sido identificado como el determinante de su crecimiento (APHAAWWA-WPCF, 1992). Los resultados obtenidos para la ciénaga Grande de Beté muestran que las concentraciones tanto de nitritos como de nitratos no sobrepasan los $0.05 \mathrm{mg} /$ 1, presentándose en forma constante en casi todas las zonas de la ciénaga con un valor de $0.02 \mathrm{mg} / 1$, a excepción de la zona central del caño donde se observa un aumento hasta de 0.05 $\mathrm{mg} / 1$ y $0.04 \mathrm{mg} / \mathrm{l}$ respectivamente como se observa en la Gráfica2.

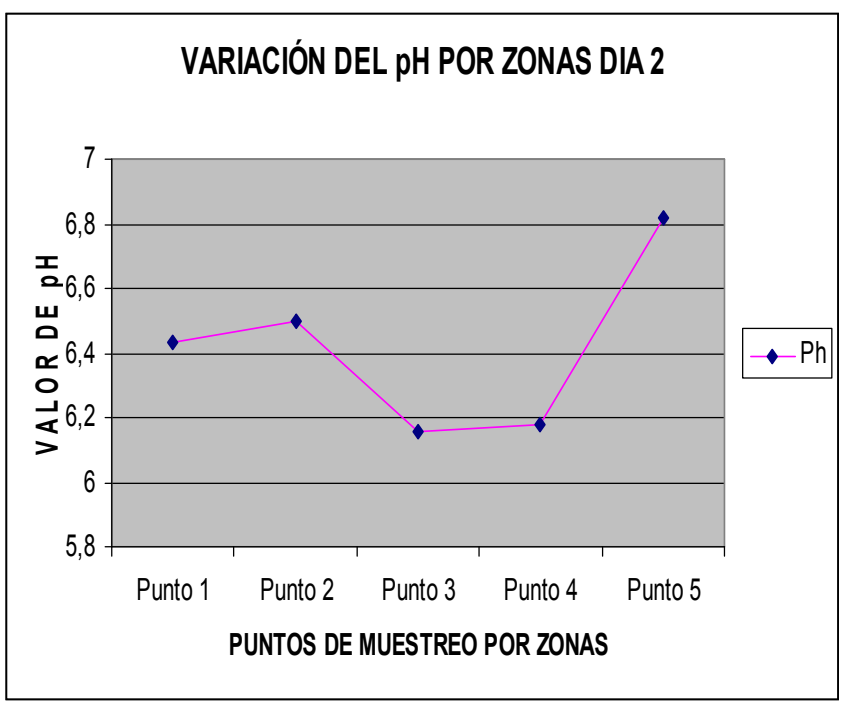

Gráfica 1. Variación de pH por zonas 
Fisicoquímica del agua y la dinámica sistémica de la ciénaga La Grande. G Ramírez-Moreno, L Vargas

Tabla 7

Variación del pH en la ciénaga Grande de Beté

\begin{tabular}{|c|c|c|c|c|c|}
\hline Día de muestreo & Punto de muestreo & Hora & $\mathbf{P h}$ & Coordenadas planas & Altura (m) \\
\hline \multirow{10}{*}{1} & 1 & $3: 10 p m$ & 6.49 & 1034095 & 78 \\
\hline & & & & 1155810 & \\
\hline & 2 & $3: 15 p m$ & 6.47 & 1034322 & 36 \\
\hline & & & & 1156804 & \\
\hline & 3 & 4:00pm & 6.54 & 1035216 & 36 \\
\hline & & & & 1157307 & \\
\hline & 4 & $4: 25 p m$ & 6.57 & 1045722 & 41 \\
\hline & & & & 1157948 & \\
\hline & 5 & $4: 35 p m$ & 6.67 & 1035893 & 40 \\
\hline & & & & 1157869 & \\
\hline \multirow{10}{*}{2} & 1 & $11: 55 \mathrm{am}$ & 6.43 & 1033941 & 40 \\
\hline & & & & 1157084 & \\
\hline & 2 & 12:05am & 6.50 & 1034205 & 36 \\
\hline & & & & 1156936 & \\
\hline & 3 & $1: 10 p m$ & 6.16 & 1034869 & 38 \\
\hline & & & & 1156787 & \\
\hline & 4 & $1: 20 p m$ & 6.18 & 1035117 & 36 \\
\hline & & & & 1157716 & \\
\hline & 5 & $1: 40 \mathrm{pm}$ & 6.82 & 1035891 & 46 \\
\hline & & & & 1157867 & \\
\hline
\end{tabular}

Tabla 8

Resultados de análisis de agua en laboratorio

\begin{tabular}{lccccc}
\hline \multicolumn{1}{c}{ Parámetro } & \multicolumn{5}{c}{ Resultados por zona } \\
& Punto $\mathbf{1}$ & Punto $\mathbf{2}$ & Punto $\mathbf{3}$ & Punto $\mathbf{4}$ & Punto 5 \\
\hline Alcalinidad (mg/l) & 10,4 & 11,4 & 12,3 & 11,2 & 9,6 \\
Nitritos (mg/l) & 0,02 & 0,02 & 0,02 & 0,04 & 0,02 \\
Dureza total (mg/l) & 40 & 30 & 26 & 10 & 40 \\
Sólidos totales (mg/l) & 390 & 96 & 112 & 236 & 200 \\
Nitratos (mg/l) & 0,02 & 0,02 & 0,02 & 0,05 & 0,02 \\
Sólidos disueltos (mg/l) & 314 & 57 & 95 & 210 & 9 \\
Sulfatos (mg/l) & 0,04 & 0,023 & 0.012 & 0,013 & 0,04 \\
Sólidos suspendidos totales (mg/l) & 76 & 39 & 17 & 26 & 191 \\
Acidez (mg/l) & 20,12 & 9,54 & 6,37 & 10,15 & 10,48 \\
Fosfatos (mg/l) & $<0,01$ & $<0,01$ & $<0,01$ & $<0,01$ & $<0,01$ \\
\hline
\end{tabular}

Los sulfatos se distribuyen ampliamente en la naturaleza y se presentan en aguas naturales como los humedales, en concentraciones que van desde pocos hasta miles de miligramos por litro, en las aguas de la ciénaga la Grande de Beté y el caño que la conecta con el río Atrato se presentan valores muy bajos desde 0,01 hasta 0,04 como se observa en 
Bioetnia Volumen 5 Nº 2 (julio-diciembre), 2008
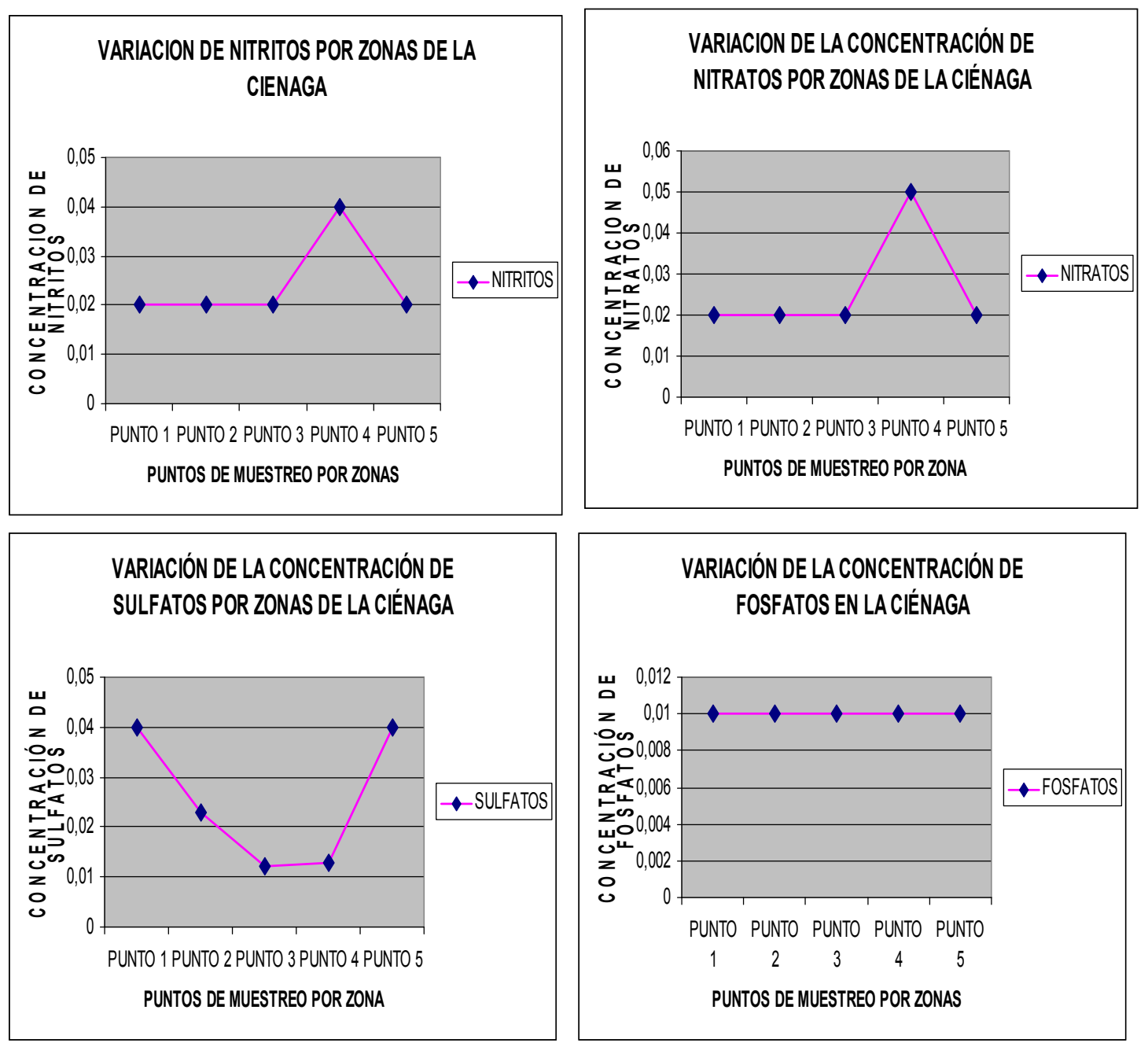

Gráfica 2. Variación de la concentración de nutrientes

la Tabla 8, presentándose las mayores concentraciones en los extremos de la ciénaga, es decir, en la zona de ribera y en la conexión entre el caño y el río (Gráfica 2).

Agentes antrópicos como los residuos del drenado de minas pueden aportar grandes cantidades de sulfatos a las aguas debido a los procesos de oxidación que se realizan durante el desarrollo de la actividad, de ahí que la concentración de sulfatos presentada sea sólo el contenido aportado de forma natural teniendo en cuenta que en la zona donde se ubica la ciénaga Grande de Beté no se lleva a cabo este tipo de extracción.

Por otro lado los fosfatos se encuentran también en las aguas naturales en soluciones, partículas, detritus o en los cuerpos de los organismos acuáticos; se forman principalmente en procesos biológicos que los aportan al agua. Con los resultados en la ciénaga Grande de Beté para este parámetro se observa que la concentración encontrada es mínima y no supera los $0.01 \mathrm{mg} / \mathrm{l}$ (Tabla 8), además es constante la distri- bución espacial del mismo en las 5 zonas definidas para el muestreo de acuerdo a la Gráfica 2.

En general todos los valores obtenidos para los nutrientes en la ciénaga Grande de Beté se consideraron bajos a pesar de que los valores de $\mathrm{pH}$ indican una acidez constante en el agua generada muy posiblemente por la descomposición de gran cantidad de materia orgánica aportada por el río, las fuertes lluvias, el lavado de los suelos y la vegetación circundante. Esto ocurre tal vez porque en los humedales que se encuentran en fases maduras de colonización, no suelen detectarse concentraciones de nutrientes muy altas en el agua, porque se encuentran atrapados en la biomasa bentónica o en el sedimento. Los nutrientes influyen en la composición de las comunidades acuáticas y éstas a su vez influyen en su concentración, estos son elementos necesarios para el crecimiento de los productores primarios.

Concentración de sólidos. La concentración de sólidos está representada en la cantidad de materiales suspendidos 


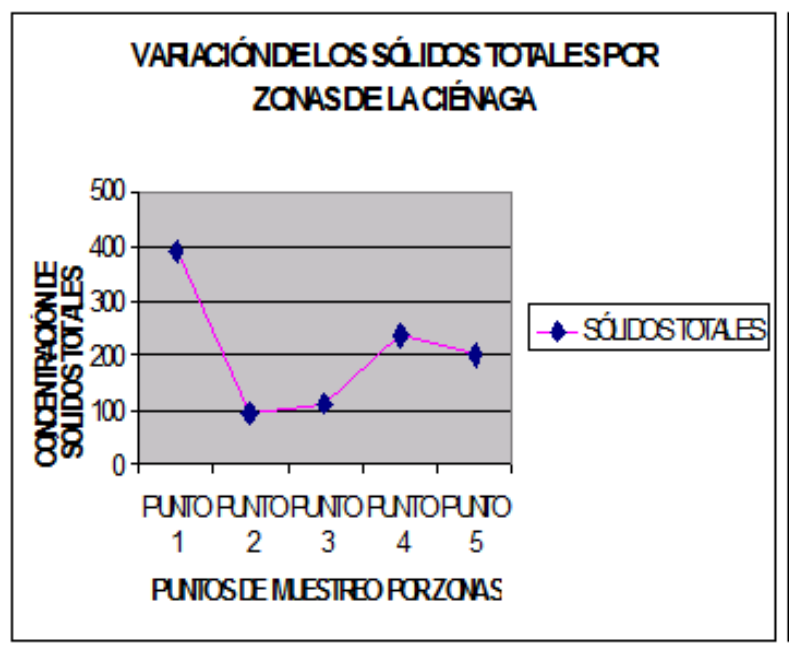

VARACÓ́NDELACONCBNTRAOÓNE SáLOS SUSTENDDOS TOTAESENLAGÉNAGA

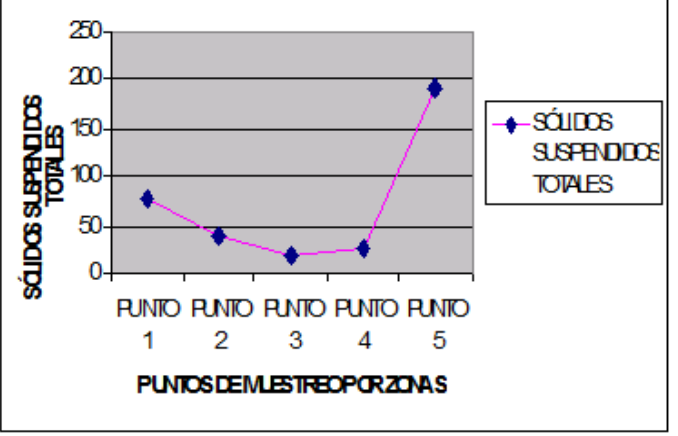

\section{Gráfica 3. Variación de la concentración de sólidos}

o disueltos en las aguas. Los sólidos totales expresan la suma de todo el material sobrenadante y el material disuelto debido a procesos de descomposición. Ambos aportan color y turbiedad a las aguas. Los resultados obtenidos para la ciénaga Grande de Beté indican que la proporción de sólidos disueltos es mucho mayor a la de sólidos suspendidos en todas las zonas de muestreo excepto en la zona 5 que conecta el río con el caño de acceso a la ciénaga (Tabla 8), situación lógica teniendo en cuenta que gran cantidad del material sobrenadante es aportado por el río a través del caño y este material sólo alcanza su mayor nivel de descomposición al ingresar a la zona central donde además recibe aportes de los suelos de la rivera de la ciénaga y la vegetación circundante y flotante que a su vez elevan los niveles de material disuelto en el agua por efecto de la descomposición.

Lo anterior se observa en la Gráfica 3 donde la variación de los sólidos totales en las 5 zonas de la ciénaga indica una mayor concentración en la zona de rivera de la ciénaga y una disminución progresiva en el recorrido hacia el río, fenómeno

\section{VARACÓ́NIELA CONGNRACÓNDE SáDOS DSUE-TOSPORZONASDELACÉNAGA}

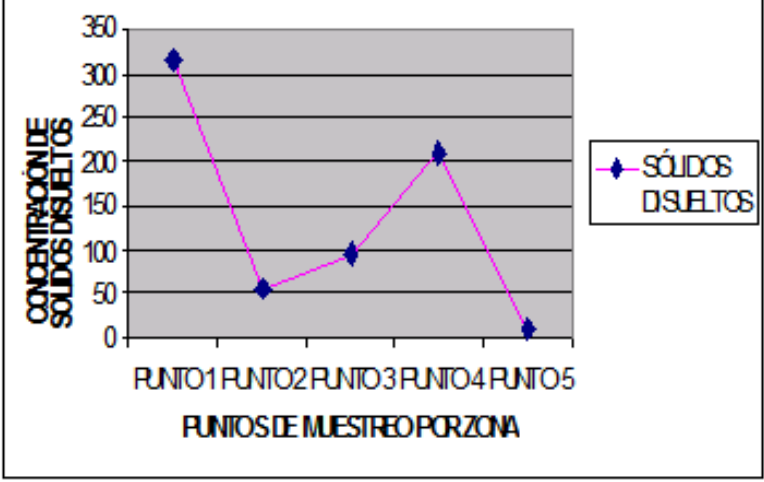

que pasa igualmente para los sólidos disueltos. Lo contrario ocurre con la variación en la concentración de sólidos suspendidos que presentan los valores más altos hacia la zona de conexión entre el caño y el río que son las zonas por donde ingresan al ecosistema, es decir, donde se encuentran en mayor cantidad arrastrados por el flujo de agua y los valores más bajos en la zona central de la ciénaga, situación que se presenta por los procesos de decantación, sedimentación y descomposición de los materiales facilitados por el flujo lento de agua, lo que no permite encontrarlos a nivel de superficie del agua.

Alcalinidad, acidezy dureza. La alcalinidad es la capacidad para neutralizar ácidos y la acidez la capacidad de neutralizar bases. La alcalinidad sirve como amortiguador de $\mathrm{pH}$ bajos o ácidos, determina la habilidad del agua para el crecimiento de algas y la vida acuática, está determinada generalmente por el contenido de carbonatos, bicarbonatos e hidróxidos y algunas sales de ácidos débiles como boratos, silicatos, nitratos y fosfatos. La alcalinidad, no sólo representa el principal sistema amortiguador del agua dulce, sino que también desempeña un papel principal en la productividad de cuerpos de agua naturales, sirviendo como una fuente de reserva para la fotosíntesis y ha sido utilizada como un indicador de la productividad de lagos. Asimismo la dureza se define como las concentraciones de calcio y magnesio ambos expresados como carbonatos.

Estos 3 parámetros se encuentran estrechamente relacionados en la composición de las aguas y la distribución espacial en la ciénaga la Grande de Beté como se muestra en la Gráfica 4. Una vez analizados estos datos y comparados con los rangos normales en aguas, se puede inferir que los valores obtenidos no afectan la estabilidad de la biota acuática en los niveles tróficos dentro del ecosistema cenagoso. El balance entre la acidez y la alcalinidad del agua se manifiesta por una leve acidez que no supera los niveles permisibles para la vida acuática y corresponde a aguas blandas o con niveles bajos 


\section{Bioetnia Volumen 5 No 2 (julio-diciembre), 2008}
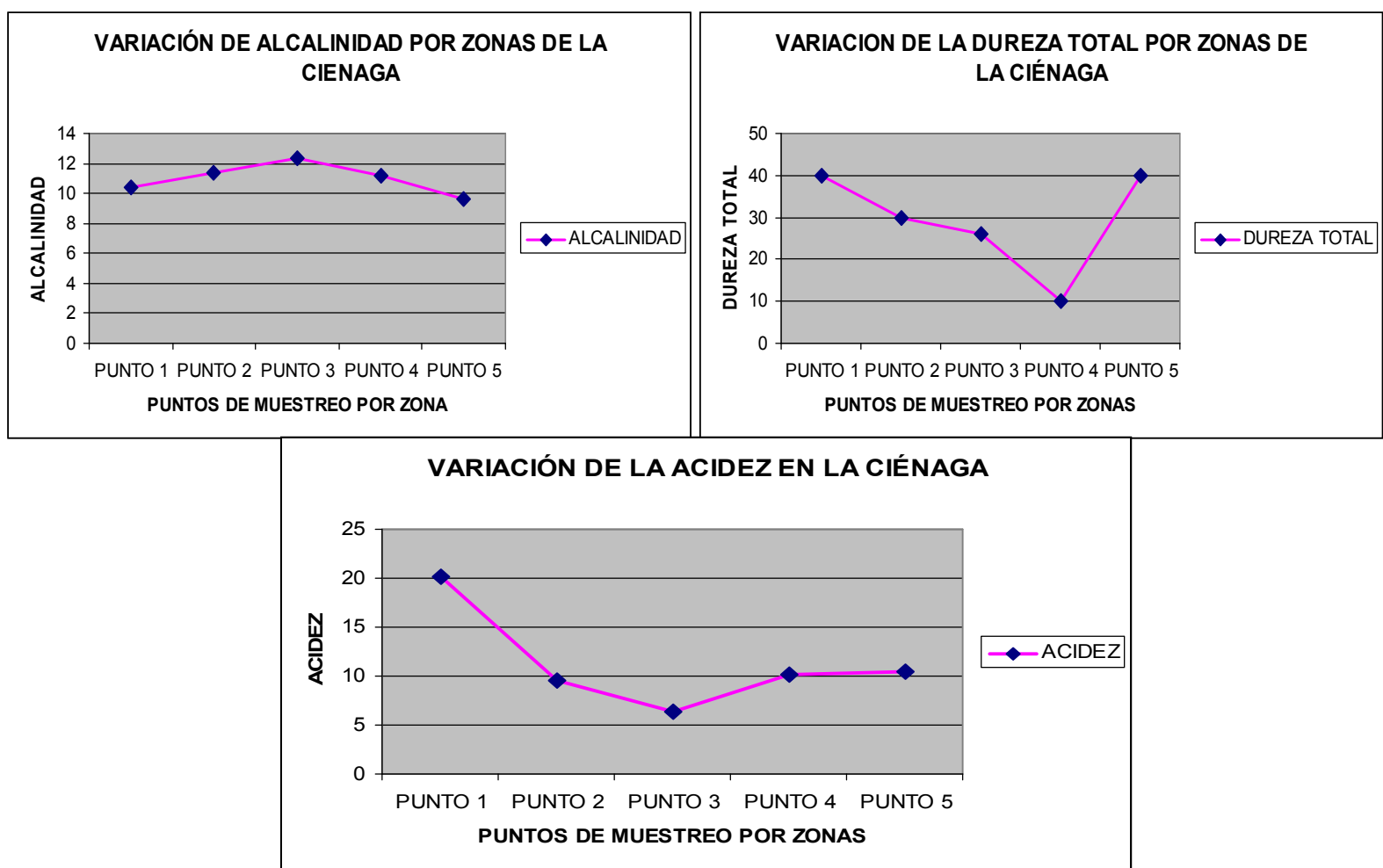

\section{Gráfica 4. Variación de la concentración de dureza, alcalinidad y acidez}

de dureza como los obtenidos.

Comparando los resultados obtenidos con los estándares internacionales de calidad de agua para la preservación de la vida acuática, se observa que el $\mathrm{pH}$ en la ciénaga Grande de Beté se encuentra dentro de los rangos permisibles establecidos, lo que indica que el recurso presenta niveles normales de acidez para el desarrollo de la biota acuática, mientras que los valores de concentración de nutrientes encontrados están muy por debajo de los niveles aceptables, fenómeno que podría estar asociado con que en este tipo de ecosistemas no suelen detectarse concentraciones de nutrientes muy altas en el agua, porque están atrapados en la biomasa bentónica o en el sedimento, como ya se había mencionado.

La caracterización ambiental preliminar de los humedales de la meseta de Popayán y Puracé en el departamento del Cauca, realizada en convenio entre la Corporación Autónoma Regional del Cauca, la WWF y la Asociación Calidris (Estela, et al., 2006), es un estudio con características muy similares al presente, donde se analizaron humedales de dos complejos entre los que se encuentran el humedal Pomona, humedal Universidad del Cauca, humedal Manzanares, humedal Genagra y el humedal Olímpica.

En el humedal la Olímpica se obtuvieron valores de $\mathrm{pH}$ de 5.85 , concentraciones de alcalinidad de $451 \mathrm{mg} / 1$, dureza 192 $\mathrm{mg} / \mathrm{l}$, nitratos $1.91 \mathrm{mg} / \mathrm{l}$, fosfatos $2.9 \mathrm{mg} / 1$, sulfatos $38 \mathrm{mg} / \mathrm{l}$, sólidos suspendidos totales de $37 \mathrm{mg} / 1$ y sólidos disueltos
$15.4 \mathrm{mg} / \mathrm{l}$ (Estela, et al., 2006). Valores que comparados con los obtenidos en el presente estudio para la ciénaga la Grande de Bete son elevados y muestran que el agua que contiene el humedal la Olímpica se encuentra absolutamente contaminada y su valor como hábitat para el mantenimiento de la biodiversidad local es limitado, lo que se explica teniendo en cuenta que los ecosistemas de la meseta de Popayán están ampliamente transformados en ambientes urbanos y rurales, por lo cual las características bióticas de sus humedales corresponden a ambientes muy perturbados. Así por ejemplo, la vegetación dominante en estos humedales se compone sobre todo de pastos propios de zonas transformadas para la ganadería extensiva, lo que indica un proceso avanzado de sucesión y/o de transformación antropogénica (Estela, et al., 2006).

También ocurre con los humedales la Pomona, Genagra y Universidad del Cauca que presentan concentraciones superiores a las encontradas en la ciénaga Grande de Beté en cuanto a parámetros de calidad del agua. Estos resultados son generados por los efectos antrópicos existentes en la gran mayoría de los ecosistemas de Popayán, ya que en ellos existen vertimientos de aguas servidas, no se detecta productividad primaria (ausencia de clorofila), se practica la ganadería extensiva y la agricultura generando escasez de vegetación (Estela, et al., 2006), lo que ocurre en un escenario contrario a la zona de estudio donde se observa la dinámica 
completa de un ecosistema de humedal con sus cadenas tróficas, la estructura completa de vegetación y la fauna que depende de ella (ictiofauna y la fauna de la zona circundante de humedal: mamíferos, reptiles, anfibios y aves), factores que están directamente relacionados con la calidad del agua presentada.

\section{CONCLUSIONESY ASPECTOS RELEVANTES}

La dinámica física y química al interior de la ciénaga está totalmente influida por la relación directa y la dinámica hídrica entre el río y la ciénaga.

Gran parte de la materia orgánica de la ciénaga es aportada por el material de arrastre de las llanuras aluviales del río Atrato y se encuentra disuelta en el agua por procesos de descomposición.

La calidad del agua encontrada muestra niveles que comparados con estándares internacionales permiten el desarrollo óptimo de la biota acuática de un ecosistema de humedal.

El estado y la dinámica del agua corresponden a la presencia y distribución del componente biológico en el ecosistema.

\section{RECOMENDACIONES PARASU CONSERVACIÓN}

Aunque el presente estudio es una aproximación al conocimiento de los humedales del Medio Atrato se recomienda implementar nuevas investigaciones que permitan conocer exactamente la biodiversidad que albergan estos ambientes y su funcionamiento para lograr la formulación de un plan eficaz de manejo y conservación.

Como medida de conservación y mantenimiento de los flujos y la dinámica hídrica propia del ecosistema se recomienda un permanente monitoreo del estado de obstrucción de los caños porque por los aportes de sólidos provenientes del río Atrato y los residuos de corte de madera generados en el caño, se presentan taponamientos que interfieren en el flujo normal del agua y por consiguiente en el transporte de nutrientes requerido para el desarrollo de este ecosistema.

También es necesario establecer control sobre el corte extractivo de madera tanto en el caño como al interior de la ciénaga porque el estado de la vegetación está directamente relacionado con la fauna presente y con el estado y la calidad del recurso agua.

Se requiere ejercer control sobre la extracción minera para evitar que los efluentes de esta actividad realizada en otras zonas sobre el río Atrato, ingresen a los ecosistemas cenagosos y contaminen el agua afectando el componente biológico y el equilibrio de dichos ecosistemas.

\section{RECOMENDACIONES COMOHERRAMIENTAEN EDUCACIÓN AMBIENTAL}

Es importante establecer una estrategia nacional, regional y local de comunicación para difundir contenidos referentes a los valores y funciones de los ecosistemas cenagosos del Medio Atrato.

Diseñar rutas de turismo ecológico alrededor de los humedales que además de concientizar a las comunidades de la importancia de los ecosistemas, les permita observar toda la biota que se desarrolla en ellos y se constituya en un mecanismo de ingreso y desarrollo para el municipio del Medio Atrato.

\section{PROYECCIONESDEINVESTIGACIÓN}

Con el presente estudio se avanza en el conocimiento requerido para la formulación de planes de manejo para los ecosistemas cenagosos del municipio Medio Atrato; no obstante dicho conocimiento se puede ampliar mediante el desarrollo de estudios que completen los resultados obtenidos, entre los cuales se pueden proyectar:

- Medición de parámetros fisicoquímicos en los diferentes estratos de la ciénaga.

- Monitoreo de contaminantes en la ciénaga asociados con la actividad minera para determinar el nivel de influencia de las actividades realizadas sobre el río Atrato al interior del ecosistema.

- Relación hidrológica entre los acuíferos y los sistemas de humedales.

- Comportamiento hídrico del ecosistema en las diferentes temporadas climáticas de la zona.

\section{LITERATURA CITADA}

APHA-AWWA-WPCF. 1992. Métodos para el análisis de aguas potables y residuales, Madrid: Editorial Diaz de Santos.

Carrizo, R. 2008. Lineamientos y metodología a aplicar para la definición de "presupuestos mínimos» en materia de control de la contaminación hídrica. Situación Ambiental de Argentina. Buenos Aires: Programa PRODIA, 2008.

Cooke, R., Griggs, J., Sánchez, L., Díaz C., Carvajal D. 2001. Recopilación y presentación de datos de recursos ambientales y culturales en la región occidental de la cuenca del canal de Panamá. Volumen 4. En: Calidad ambiental. Informe final de la región occidental de la cuenca del Canal, Consorcio TLBG UP STRI, Panamá.

Estela, F., Hernández, C., Eusse, D., Artunduaga, D. 2006. Caracterización ambiental preliminar de los humedales de la meseta de Popayán y Puracé en el departamento del Cauca. Cali: CRC-WWF-CALIDRIS, 2006.

Ministerio de Vivienda, Ordenamiento Territorial y Medio Ambiente de Uruguay. 2007. La calidad del agua del río Uruguay, resultados de las tres primeras campañas. Dirección Nacional de Medio Ambiente. 